\section{Calcium Ameliorates the Tolerance of Lisianthus [Eustoma grandiflorum (Raf.) Shinn.] to Alkalinity in Irrigation Water}

\author{
Lucina Gómez-Pérez, Luis Alonso Valdez-Aguilar'1, \\ Alberto Sandoval-Rangel, Adalberto Benavides-Mendoza, \\ and Rosalinda Mendoza-Villarreal \\ Departamento de Horticultura, Universidad Autónoma Agraria Antonio \\ Narro, Calzada Antonio Narro 1923, Buenavista, Saltillo, Coah., México \\ 25315

\section{Ana María Castillo-González \\ Instituto de Horticultura. Universidad Autónoma Chapingo, Carr. México- Texcoco km. 36.5, Chapingo, México 56230}

Additional index words. antioxidant system, bicarbonate, ornamental plants, plant nutrition, soilless culture

\begin{abstract}
Irrigation water high in alkalinity can severely compromise the growth and marketability of ornamental plants. In the present study we investigated the response of lisianthus to increased calcium (Ca) when irrigated with solutions containing high levels of bicarbonate $\left(\mathrm{HCO}_{3}{ }^{-}\right)$-induced alkalinity. Alkalinity in irrigation water reduced the growth of lisianthus; however, plants supplemented with an increased concentration of Ca at alkalinity levels from 4 to $7 \mathrm{meq} \cdot \mathrm{L}^{-1}$ of $\mathrm{HCO}_{3}^{-}$exhibited improved growth and dry mass (DM) accumulation or were not detrimentally affected, demonstrating that Ca contributes to the increase of the tolerance of lisianthus to alkalinity. Supplementary Ca did maintain a high stomatal conductance $\left(g_{S}\right)$ and transpiration rate when alkalinity was at $4 \mathrm{meq} \cdot \mathrm{L}^{-1}$, which explained the lower water potential in young leaves. Plants irrigated with solutions containing supplementary Ca had higher total DM, which was associated with a higher $g_{\mathrm{S}}$; however, when conductance was higher than $0.280 \mathrm{~cm} \cdot \mathrm{s}^{-1}$, like in plants with no supplementary Ca, DM tended to decrease. At a typical Ca concentration, there was a disruption on stomata functioning as $g_{\mathrm{S}}$ and transpiration rate increased, which was associated with a reduction in shoot potassium (K). Calcium ameliorated the uptake of $K$ when alkalinity was $4 \mathrm{meq} \cdot \mathrm{L}^{-1}$ by allowing a less marked reduction in shoot $K$ concentration. Chlorophyll was reduced by increasing alkalinity as a result of a decrease in shoot iron (Fe); however, supplementary $\mathrm{Ca}$ also contributed in increasing plant tolerance to alkalinity at $4 \mathrm{meq} \cdot \mathrm{L}^{-1}$ by sustaining a high shoot Fe concentration. Supplementary Ca increased catalase and peroxidase activities, indicating that lisianthus responded to the stress by enhancing the activity of these enzymes to reduce oxidative damage.
\end{abstract}

Alkalinity affects plant production in the arable lands of the arid and semiarid regions of the world. Irrigation water of high alkalinity may cause nutritional problems in cultivated horticultural plants, specifically chlorosis in young leaves resulting from the formation of Fe forms that are unavailable for plant uptake. Alkalinity may also be associated with deficiency of cooper $(\mathrm{Cu})$, zinc $(\mathrm{Zn}), \mathrm{K}$, and phosphorus $(\mathrm{P})$ resulting from low solubility (FAO, 2000).

Alkalinity in water is caused mainly by carbonate $\left(\mathrm{CO}_{3}{ }^{2-}\right)$ and $\mathrm{HCO}_{3}{ }^{-}$, which at high concentrations are detrimental for plant growth (Cartmill et al., 2007). Use of irrigation water of high alkalinity leads to increased $\mathrm{pH}$ of the

Received for publication 25 Nov. 2013. Accepted for publication 26 Mar. 2014.

${ }^{1}$ To whom reprint request should be addressed; e-mail luisalonso.valdez@uaaan.mx. regulating the response reactions and developmental processes (Steinhorst and Kudla, 2013). However, there is limited information on the potential role of $\mathrm{Ca}$ in enhancing plant tolerance to alkalinity stress. Calcium is a structural element that constitutes the middle lamellae, wall, and membranes of plant cells; participates in cell division, extension, and compartmentalization; and regulates the action of hormones and signals (Marschner, 1995). Supplementation with increasing $\mathrm{Ca}$ has been suggested to increase plant tolerance to salinity stress (Epstein, 1998). Kaya et al. (2002) and Tuna et al. (2007) reported that increased $\mathrm{Ca}$ concentrations resulted in enhanced growth and yield of strawberry (Fragaria xananassa Duch.) and tomato (Solanum lycopersicon L.) plants, respectively, exposed to high salinity conditions. The present study was conducted to investigate if supplementary $\mathrm{Ca}$ enhances the response of lisianthus to high levels of $\mathrm{HCO}_{3}^{-}$-induced alkalinity.

\section{Materials and Methods}

Cultural conditions and plant material. The experiment was conducted in a greenhouse located at Universidad Autónoma Agraria Antonio Narro, in north México (lat. $25^{\circ} 27^{\prime} \mathrm{N}$, long. $101^{\circ} 02^{\prime} \mathrm{W}, 1610 \mathrm{~m}$ above sea level). Average maximum/minimum temperature were $33 / 14{ }^{\circ} \mathrm{C}$, and maximum and minimum relative humidity for experiment duration averaged $77 \%$ and $64 \%$, respectively. Average photosynthetically active radiation $(P A R)$ measured at solar noon was $361 \mu \mathrm{mol} \cdot \mathrm{m}^{-2} \cdot \mathrm{s}^{-1}$.

Lisianthus cv. ABC Blue plants were transplanted (22 Mar. 2013) into 40-L rigid plastic containers (10 plants per container) with a drainage hole for retrieval of the nutrient solution. Each container was considered an experimental unit and contained $35 \mathrm{~L}$ of horticultural-grade perlite [33\% waterholding capacity $(\mathrm{v} / \mathrm{v}), 64 \%$ air-filled pore space, $0.25 \mathrm{~g} \cdot \mathrm{cm}^{-3}$ apparent density].

Nutrient solution application. Experimental units were fertigated with nutrient solutions with increasing $\mathrm{HCO}_{3}{ }^{-}$-induced alkalinity concentrations: $1,4,7$ and $10 \mathrm{meq} \cdot \mathrm{L}^{-1}$ of $\mathrm{HCO}_{3}{ }^{-}$(from $\mathrm{KHCO}_{3}$ and $\mathrm{NaHCO}_{3}$ ) and two concentrations of $\mathrm{Ca}$ : control $\left(8 \mathrm{meq} \cdot \mathrm{L}^{-1}\right)$ and supplementary $\left(11 \mathrm{meq} \cdot \mathrm{L}^{-1}\right)$. Alkalinity was measured by titration three times a week and concentration of $\mathrm{HCO}_{3}^{-}$adjusted accordingly by adding $\mathrm{NaHCO}_{3}$. The remaining nutrients were supplied according to Steiner's nutrient solution formulation (Steiner, 1984), including macronutrients (meq. $\mathrm{L}^{-1}$ ): $12 \mathrm{NO}_{3}{ }^{-}$, $1 \mathrm{H}_{2} \mathrm{PO}_{4}^{-}, 7 \mathrm{~K}, 4$ magnesium $(\mathrm{Mg}), 2 \mathrm{SO}_{4}{ }^{2-}$, and micronutrients $\left(\mathrm{mg} \cdot \mathrm{L}^{-1}\right): 5.3 \mathrm{Fe}, 0.4 \mathrm{Zn}$, 2.6 manganese, $0.5 \mathrm{Cu}, 0.2$ boron (B), and 0.2 molybdenum. Solution $\mathrm{pH}$ for solutions with 1, 4, 7, and $10 \mathrm{meq} \cdot \mathrm{L}^{-1} \mathrm{HCO}_{3}^{-}$was 6.5, 7.2, 7.7 , and 8.5 , respectively, whereas electrical conductivity for control and supplementary Ca solutions was 2, 2.3, 2.5, and 2.7 and 2.2, $2.5,2.6$, and $2.9 \mathrm{dS} \cdot \mathrm{m}^{-1}$, respectively. Fertigation was applied through a drip irrigation system with eight emitters per experimental 
unit, and leachate was collected for reuse. Plants were irrigated hourly for $3 \mathrm{~min}(\approx 1.0 \mathrm{~L})$ from $0800 \mathrm{AM}$ to $1800 \mathrm{HR}$. Evapotranspirated water was replenished daily to each stock tank and the nutrient solutions were replaced every $10 \mathrm{~d}$.

Plant growth and nutrient status. At experiment termination, $90 \mathrm{~d}$ after transplanting, growth measurements were recorded in all the plants from each experimental unit, including shoot length (substrate to the top of the inflorescence), diameter at the base of the stem, diameter of flowers (third fully expanded flower), and number of flowers. The plants were separated into roots, stems, leaves, and flowers; washed twice with distilled water; and placed in an oven at $70{ }^{\circ} \mathrm{C}$ for $72 \mathrm{~h}$. DM was recorded for each plant part.

Dry shoot tissues were ground to pass a 40-mesh sieve (A-10; Tekmar, IKA Labortechnik, Germany) and digested in a 2:1 mixture of $\mathrm{H}_{2} \mathrm{SO}_{4}: \mathrm{HClO}_{4}$ and $2 \mathrm{~mL}$ of $30 \% \mathrm{H}_{2} \mathrm{O}_{2}$. The digested samples were analyzed for nitrogen $(\mathrm{N})$ with micro-Kjeldahl procedure, whereas $\mathrm{K}, \mathrm{P}, \mathrm{Ca}, \mathrm{Mg}, \mathrm{Cu}, \mathrm{Fe}$, and $\mathrm{Zn}$ were analyzed with an inductively coupled plasma emission spectrometer (ICP-AES, model Liberty; VARIAN, Santa Clara, CA).

Photosynthetic parameters, chlorophyll concentration, leaf water potential, and enzymatic activity. Net photosynthetic rate, $g_{\mathrm{S}}$, and transpiration rate were measured (LI-COR, Inc., Lincoln, NE) at noon on young leaves (the third fully developed leaf from top to bottom) of 90-d-old plants. Average PAR, $\mathrm{CO}_{2}$ concentration, and temperature were $596 \mu \mathrm{mol} \cdot \mathrm{m}^{-2} \cdot \mathrm{s}^{-1}, 364 \mathrm{ppm}$, and $28.9^{\circ} \mathrm{C}$, respectively. Three measurements on each leaf from one plant per experimental unit were recorded.

Photosynthetic pigments (chlorophyll a, b, and $\mathrm{a}+\mathrm{b}$ ) and catalase and peroxidase activities were measured in young leaves of three plants per replication as described by Jeffrey and Humphrey (1975), Masia (1998), and Kar and Mishra (1976), respectively, at experiment termination. Water potential $\left(\Psi_{\mathrm{w}}\right)$ of one young (top third of the plant) and one mature (bottom third of the plant) leaf sampled at solar noon (1200 to $1300 \mathrm{HR}$ ) was measured (Scholander Pressure Chamber; Soil Moisture Equipment Corp., Santa Barbara, CA) at experiment termination.

Statistical design. Three replicates of each experimental unit (container with 10 plants) were distributed in a factorial complete randomized block design with four levels of $\mathrm{HCO}_{3}{ }^{-}$and two levels of $\mathrm{Ca}$. Data were analyzed using analysis of variance and linear, quadratic, or cubic trend analysis with SAS (SAS Version 8.0; SAS Institute, Cary, NC).

\section{Results}

Plant growth. Plants with control Ca concentration exhibited a linear decrease with increasing alkalinity in shoot length (Fig. 1A) and diameter (Table 1), whereas plants with supplemental Ca showed a quadratic response, indicating that supplementary $\mathrm{Ca}$ improved plant growth (length) or was unaffected (diameter) even at an alkalinity of $4 \mathrm{meq} \cdot \mathrm{L}^{-1}$. The

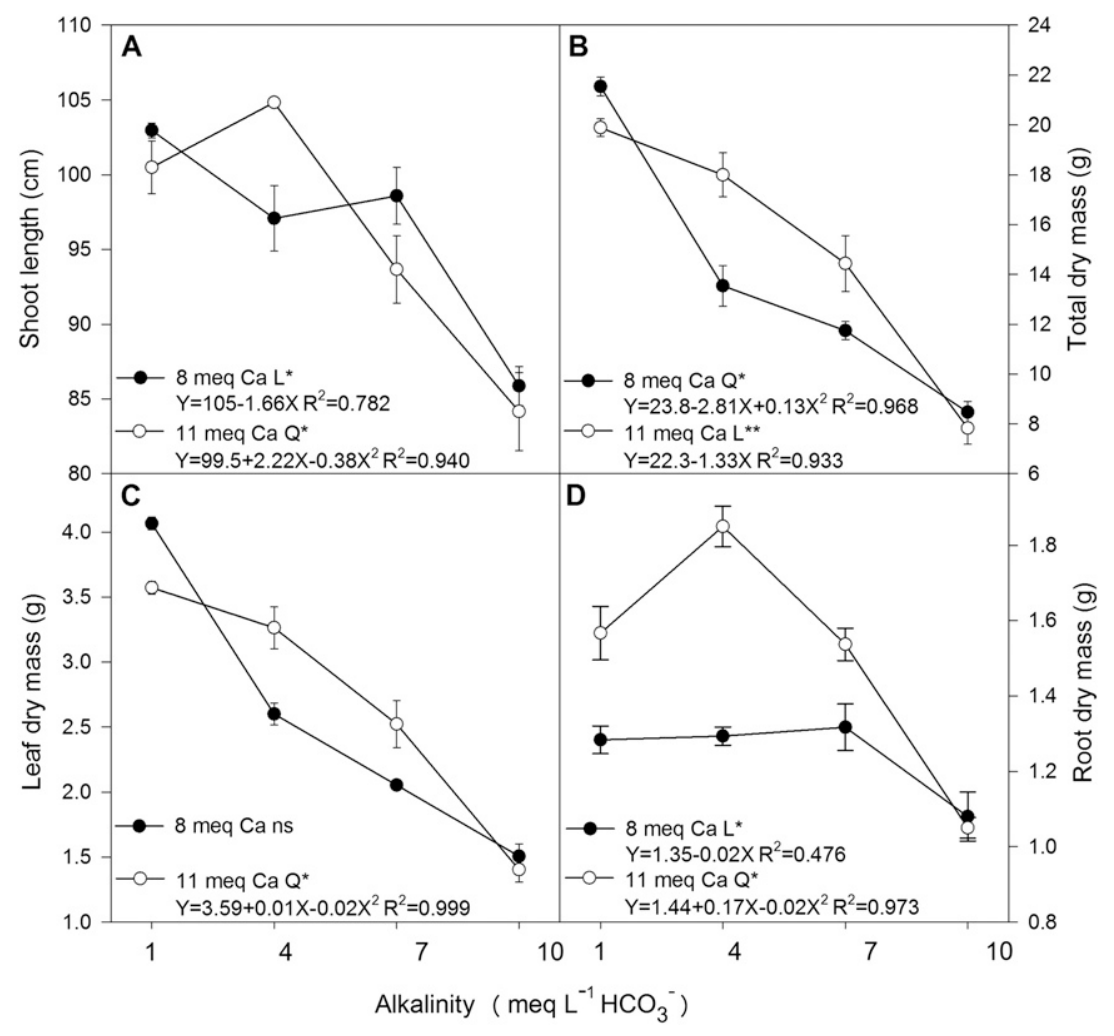

Fig. 1. (A-D) Effect of supplementary calcium $(\mathrm{Ca})$ and bicarbonate $\left(\mathrm{HCO}_{3}{ }^{-}\right)$-induced alkalinity in the nutrient solution on growth parameters of lisianthus [Eustoma grandiflorum (Raf.) Shinn.] cv. ABC Blue at experiment termination (90 d after transplanting). Bars represent the SEM $(\mathrm{n}=3)$. L, Q, linear, quadratic trends, respectively; NS, *,** nonsignificant and significant at $P<0.05$ and $<0.01$, respectively. Alkalinity effect significant for shoot length $(P<0.001)$, total dry mass $(P<0.001)$, leaf dry mass $(P<0.001)$, and root dry mass $(P=0.002)$. Calcium effect significant for root dry mass $(P=$ $0.004)$. Interaction effect significant for total dry mass $(P=0.044)$ and leaf dry mass $(P=0.033)$.

number of flower buds was decreased; however, the decrease was less pronounced in plants with increased $\mathrm{Ca}$ because flower bud count was $12 \%$ and $34 \%$ lower when alkalinity was increased to 4 meq. $\mathrm{L}^{-1}$ in Ca-supplemented and control plants, respectively (Table 1 ). The diameter of flowers did not exhibit a consistent tendency (Table 1); however, plants with supplementary Ca produced flowers of similar or larger size than those of the control plants when alkalinity was $7 \mathrm{meq} \cdot \mathrm{L}^{-1}$ or less.

Total DM of plants (Fig. 1B) as well as leaf (Fig. 1C), stem (Table 1), and flower DM (Table 1) were reduced by increasing alkalinity; however, the deleterious effect of high $\mathrm{HCO}_{3}{ }^{-}$was less pronounced in plants that received supplementary $\mathrm{Ca}$ when alkalinity was 4 to $7 \mathrm{meq} \cdot \mathrm{L}^{-1}$. Alkalinity had no effect on root DM of control plants (Fig. 1D), whereas supplementary $\mathrm{Ca}$ increased root biomass accumulation regardless of the alkalinity level, except when $\mathrm{HCO}_{3}{ }^{-}$was at $10 \mathrm{meq} \cdot \mathrm{L}^{-1}$.

Physiological parameters. Increasing alkalinity linearly reduced photosynthetic rate of lisianthus regardless of $\mathrm{Ca}$ concentration in the nutrient solution (Table 2). Transpiration rate and $g_{\mathrm{S}}$ were unaffected by alkalinity in plants with control Ca concentration; however, in plants with supplementary $\mathrm{Ca}$, transpiration rate increased with $4 \mathrm{meq} \cdot \mathrm{L}^{-1}$ of $\mathrm{HCO}_{3}{ }^{-}$and conductance decreased with increasing concentrations of $\mathrm{HCO}_{3}^{-}$(Table 2).
Plants irrigated with supplementary $\mathrm{Ca}$ exhibited an association between total DM and $g_{\mathrm{S}}$ [total DM $\left.=202\left(g_{\mathrm{S}}\right)-34.6 ; R^{2}=0.999\right]$; however, in plants with control Ca concentration, total DM accumulation tended to decrease [total DM $=-535\left(g_{\mathrm{S}}\right)+162, R^{2}=$ $0.856]$. In control plants, $\psi_{\mathrm{w}}$ increased in young and mature leaves when exposed to the highest alkalinity (10 meq. $\mathrm{L}^{-1}$ ) (Table 2$)$; however, in plants with supplementary $\mathrm{Ca}$, an initial decrease in $\psi_{\mathrm{w}}$ of both young and mature leaves was detected when exposed to $4 \mathrm{meq} \cdot \mathrm{L}^{-1}$ of $\mathrm{HCO}_{3}^{-}$, which was restored when alkalinity concentration was increased to 7 and $10 \mathrm{meq} \cdot \mathrm{L}^{-1}$. Increasing alkalinity was associated with a decrease in photosynthetic pigments regardless of $\mathrm{Ca}$ concentration (Table 3 ) and induced an increase in the activity of catalase (Fig. 2A) and peroxidase (Fig. 2B) at both $\mathrm{Ca}$ concentrations; however, enzyme activity was higher in plants that received supplementary $\mathrm{Ca}$, especially peroxidase activity, at high alkalinity.

Nutrient status. In both control and plants with supplementary $\mathrm{Ca}$, increasing alkalinity caused a decrease in shoot $\mathrm{K}, \mathrm{Ca}$ (Table 4), $\mathrm{Zn}$, and Fe (Table 5) concentration, whereas shoot $\mathrm{N}$ was affected only in control plants (Table 4). Plants receiving supplementary $\mathrm{Ca}$ had a higher concentration of $\mathrm{N}, \mathrm{K}, \mathrm{Ca}$ (Table 4 ), and Fe (Table 5) when alkalinity of the nutrient solution was $4 \mathrm{meq} \cdot \mathrm{L}^{-1}$. 
Table 1. Effect of calcium $(\mathrm{Ca})$ concentration and bicarbonate $\left(\mathrm{HCO}_{3}{ }^{-}\right)$-induced alkalinity in the nutrient solution on growth parameters $($mean \pm SE, $\mathrm{n}=3)$ of lisianthus [Eustoma grandiflorum (Raf.) Shinn.] cv. ABC Blue at experiment termination (90 d after transplanting).

\begin{tabular}{|c|c|c|c|c|c|c|c|c|c|c|}
\hline \multirow[b]{3}{*}{$\mathrm{HCO}_{3}{ }^{-}\left(\mathrm{meq} \cdot \mathrm{L}^{-1}\right)$} & \multicolumn{2}{|c|}{ Stem diam $(\mathrm{mm})$} & \multicolumn{2}{|c|}{ Flower bud number } & \multicolumn{2}{|c|}{ Flower diam $(\mathrm{cm})$} & \multicolumn{2}{|c|}{ Stem dry mass $(\mathrm{g})$} & \multicolumn{2}{|c|}{ Flower dry mass (g) } \\
\hline & \multicolumn{10}{|c|}{$\mathrm{Ca}\left(\mathrm{meq} \cdot \mathrm{L}^{-1}\right)$} \\
\hline & 8 & 11 & 8 & 11 & 8 & 11 & 8 & 11 & 8 & 11 \\
\hline 1 & $7.07 \pm 0.17$ & $6.94 \pm 0.05$ & $15.3 \pm 0.94$ & $13.8 \pm 0.94$ & $100.9 \pm 3.20$ & $99.0 \pm 3.55$ & $9.25 \pm 0.30$ & $8.53 \pm 0.91$ & $6.93 \pm 0.61$ & $6.22 \pm 0.15$ \\
\hline 4 & $6.29 \pm 0.30$ & $6.68 \pm 0.09$ & $10.1 \pm 1.17$ & $12.2 \pm 1.04$ & $91.9 \pm 0.79$ & $98.8 \pm 6.19$ & $5.50 \pm 0.75$ & $7.54 \pm 0.37$ & $4.14 \pm 0.93$ & $5.33 \pm 0.16$ \\
\hline 7 & $5.79 \pm 0.06$ & $5.87 \pm 0.43$ & $8.08 \pm 0.46$ & $10.9 \pm 2.19$ & $100.5 \pm 2.80$ & $100.4 \pm 4.90$ & $4.66 \pm 0.24$ & $5.69 \pm 0.93$ & $3.71 \pm 0.56$ & $4.68 \pm 0.14$ \\
\hline 10 & $4.71 \pm 0.20$ & $4.67 \pm 0.37$ & $6.08 \pm 0.73$ & $6.25 \pm 1.39$ & $95.2 \pm 4.37$ & $88.1 \pm 1.72$ & $3.19 \pm 0.44$ & $3.08 \pm 0.67$ & $2.69 \pm 0.49$ & $2.29 \pm 0.49$ \\
\hline Trend analysis ${ }^{z}$ & $\mathrm{~L}^{* *}$ & $\mathrm{Q}^{*}$ & $\mathrm{Q}^{*}$ & $\mathrm{Q}^{*}$ & $\mathrm{C}^{*}$ & $\mathrm{~L}^{*}$ & $\mathrm{Q}^{*}$ & $Q^{*}$ & $\mathrm{~L}^{* *}$ & $\mathrm{~L}^{* *}$ \\
\hline \multicolumn{11}{|c|}{ Analysis of variance } \\
\hline $\mathrm{HCO}_{3}^{-}$ & \multicolumn{2}{|c|}{$P<0.001$} & \multicolumn{2}{|c|}{$P<0.001$} & \multicolumn{2}{|c|}{$P=0.005$} & \multicolumn{2}{|c|}{$P<0.001$} & \multicolumn{2}{|c|}{$P<0.001$} \\
\hline $\mathrm{Ca}$ & \multicolumn{2}{|c|}{ NS } & \multirow{2}{*}{\multicolumn{2}{|c|}{$\begin{array}{c}\text { NS } \\
P=0.048\end{array}$}} & \multirow{2}{*}{\multicolumn{2}{|c|}{$\begin{array}{c}\text { NS } \\
P=0.047\end{array}$}} & \multicolumn{2}{|c|}{ NS } & \multicolumn{2}{|c|}{ NS } \\
\hline Interaction & \multicolumn{2}{|c|}{$P=0.005$} & & & & & \multicolumn{2}{|c|}{$P=0.049$} & \multicolumn{2}{|c|}{ NS } \\
\hline
\end{tabular}

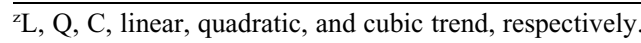

*,** Significant at $P<0.05$ and $<0.01$, respectively.

NS $=$ nonsignificant.

Table 2. Effect of calcium $(\mathrm{Ca})$ concentration and bicarbonate $\left(\mathrm{HCO}_{3}{ }^{-}\right)$-induced alkalinity in the nutrient solution on selected physiological and water potential $\left(\psi_{\mathrm{w}}\right)$ parameters (mean $\pm \mathrm{sE}, \mathrm{n}=3$ ) of lisianthus [Eustoma grandiflorum (Raf.) Shinn.] cv. ABC Blue at experiment termination ( $90 \mathrm{~d}$ after transplanting).

\begin{tabular}{|c|c|c|c|c|c|c|c|c|c|c|}
\hline \multirow{3}{*}{$\begin{array}{l}\mathrm{HCO}_{3}^{-} \\
\left(\mathrm{meq} \cdot \mathrm{L}^{-1}\right)\end{array}$} & \multicolumn{2}{|c|}{$\begin{array}{l}\text { Photosynthetic rate } \\
\left(\mu \mathrm{mol} \mathrm{CO}_{2} \mathrm{~m}^{-2} \cdot \mathrm{s}^{-1}\right)\end{array}$} & \multicolumn{2}{|c|}{$\begin{array}{l}\text { Transpiration rate } \\
\left(\mu \mathrm{g} \cdot \mathrm{cm}^{-2} \cdot \mathrm{s}^{-1}\right)\end{array}$} & \multicolumn{2}{|c|}{$\begin{array}{l}\text { Stomatal conductance } \\
\left(\mathrm{cm} \cdot \mathrm{s}^{-1}\right)\end{array}$} & \multicolumn{2}{|c|}{ Young leaf $\psi_{\mathrm{w}}(\mathrm{MPa})$} & \multicolumn{2}{|c|}{ Mature leaf $\psi_{\mathrm{w}}(\mathrm{MPa})$} \\
\hline & \multicolumn{10}{|c|}{$\mathrm{Ca}\left(\mathrm{meq} \cdot \mathrm{L}^{-1}\right)$} \\
\hline & 8 & 11 & 8 & 11 & 8 & 11 & 8 & 11 & 8 & 11 \\
\hline 1 & $12.3 \pm 0.65$ & $12.3 \pm 0.50$ & $9.45 \pm 0.06$ & $9.09 \pm 0.19$ & $0.263 \pm 0.01$ & $0.270 \pm 0.03$ & $-0.926 \pm 0.01$ & $-0.910 \pm 0.01$ & $-0.910 \pm 0.00$ & $-0.873 \pm 0.01$ \\
\hline 4 & $12.8 \pm 0.26$ & $10.3 \pm 0.57$ & $9.46 \pm 0.65$ & $9.69 \pm 0.11$ & $0.283 \pm 0.03$ & $0.260 \pm 0.05$ & $-0.903 \pm 0.01$ & $-0.933 \pm 0.00$ & $-0.893 \pm 0.01$ & $-0.923 \pm 0.01$ \\
\hline 7 & $10.3 \pm 0.41$ & $9.63 \pm 0.28$ & $9.96 \pm 0.41$ & $9.31 \pm 0.96$ & $0.280 \pm 0.03$ & $0.243 \pm 0.05$ & $-0.923 \pm 0.01$ & $-0.900 \pm 0.01$ & $-0.913 \pm 0.01$ & $-0.890 \pm 0.02$ \\
\hline 10 & $8.83 \pm 0.11$ & $7.16 \pm 0.11$ & $9.99 \pm 0.39$ & $9.03 \pm 0.37$ & $0.283 \pm 0.01$ & $0.210 \pm 0.03$ & $-0.856 \pm 0.01$ & $-0.873 \pm 0.01$ & $-0.883 \pm 0.01$ & $-0.893 \pm 0.01$ \\
\hline Trend analysis ${ }^{z}$ & $\mathrm{~L}^{*}$ & $\mathrm{~L}^{*}$ & NS & $\mathrm{Q}^{*}$ & NS & $\mathrm{L}^{*}$ & $\mathrm{C}^{* *}$ & $\mathrm{Q}^{*}$ & NS & $C^{*}$ \\
\hline \multicolumn{11}{|c|}{ Analysis of variance } \\
\hline $\mathrm{HCO}_{3}^{-}$ & \multicolumn{2}{|c|}{$P=0.004$} & \multicolumn{2}{|c|}{ NS } & \multicolumn{2}{|c|}{ NS } & \multicolumn{2}{|c|}{$P<0.001$} & \multicolumn{2}{|c|}{ NS } \\
\hline $\mathrm{Ca}$ & \multicolumn{2}{|c|}{ NS } & \multicolumn{2}{|c|}{$P=0.049$} & \multicolumn{2}{|c|}{$P=0.050$} & \multirow{2}{*}{\multicolumn{2}{|c|}{$\begin{array}{c}\text { NS } \\
P=0.016\end{array}$}} & \multicolumn{2}{|c|}{ NS } \\
\hline Interaction & \multicolumn{2}{|c|}{ NS } & \multicolumn{2}{|c|}{ NS } & \multicolumn{2}{|c|}{ NS } & & & \multicolumn{2}{|c|}{$P=0.042$} \\
\hline
\end{tabular}

${ }^{\mathrm{z}} \mathrm{L}, \mathrm{Q}, \mathrm{C}$, linear, quadratic, and cubic trend, respectively.

NS, *, ** Nonsignificant and significant at $P<0.05$ and $<0.01$, respectively.

Table 3. Effect of calcium $(\mathrm{Ca})$ concentration and bicarbonate $\left(\mathrm{HCO}_{3}{ }^{-}\right)$-induced alkalinity in the nutrient solution on chlorophyll concentration $(\mathrm{mean} \pm$ sE, $\mathrm{n}=3)$ in young leaves of lisianthus [Eustoma grandiflorum (Raf.) Shinn.] cv. ABC Blue at experiment termination (90 d after transplanting).

\begin{tabular}{|c|c|c|c|c|c|c|}
\hline \multirow[b]{3}{*}{$\mathrm{HCO}_{3}{ }^{-}\left(\mathrm{meq} \cdot \mathrm{L}^{-1}\right)$} & \multicolumn{2}{|c|}{ Chlorophyll $\mathrm{a}+\mathrm{b}\left(\mathrm{mg} \cdot \mathrm{g}^{-1}\right)$} & \multicolumn{2}{|c|}{ Chlorophyll a $\left(\mathrm{mg} \cdot \mathrm{g}^{-1}\right)$} & \multicolumn{2}{|c|}{ Chlorophyll b $\left(\mathrm{mg} \cdot \mathrm{g}^{-1}\right)$} \\
\hline & \multicolumn{6}{|c|}{$\mathrm{Ca}\left(\mathrm{meq} \cdot \mathrm{L}^{-1}\right)$} \\
\hline & 8 & 11 & 8 & 11 & 8 & 11 \\
\hline 1 & $1.24 \pm 0.09$ & $1.21 \pm 0.10$ & $0.48 \pm 0.04$ & $0.47 \pm 0.03$ & $0.77 \pm 0.04$ & $0.75 \pm 0.05$ \\
\hline 4 & $1.19 \pm 0.07$ & $1.08 \pm 0.07$ & $0.48 \pm 0.04$ & $0.39 \pm 0.03$ & $0.72 \pm 0.07$ & $0.75 \pm 0.04$ \\
\hline 7 & $0.94 \pm 0.03$ & $0.99 \pm 0.10$ & $0.33 \pm 0.01$ & $0.36 \pm 0.04$ & $0.61 \pm 0.02$ & $0.64 \pm 0.05$ \\
\hline 10 & $0.80 \pm 0.04$ & $0.80 \pm 0.07$ & $0.28 \pm 0.02$ & $0.27 \pm 0.02$ & $0.52 \pm 0.02$ & $0.53 \pm 0.04$ \\
\hline Trend analysis ${ }^{\mathrm{z}}$ & $\mathrm{L}^{* *}$ & $\mathrm{~L}^{*}$ & $\mathrm{~L}^{* *}$ & $\mathrm{~L}^{* *}$ & $\mathrm{~L}^{* *}$ & $\mathrm{~L}^{*}$ \\
\hline \multicolumn{7}{|c|}{ Analysis of variance } \\
\hline $\mathrm{HCO}_{3}^{-}$ & \multicolumn{2}{|c|}{$P<0.001$} & \multicolumn{2}{|c|}{$P<0.001$} & \multicolumn{2}{|c|}{$P<0.001$} \\
\hline $\mathrm{Ca}$ & \multicolumn{2}{|c|}{ NS } & \multicolumn{2}{|c|}{ NS } & \multicolumn{2}{|c|}{ NS } \\
\hline Interaction & \multicolumn{2}{|c|}{ NS } & \multicolumn{2}{|c|}{ NS } & \multicolumn{2}{|c|}{ NS } \\
\hline
\end{tabular}

${ }^{\mathrm{z}} \mathrm{L}, \mathrm{Q}, \mathrm{C}$, linear, quadratic, and cubic trend, respectively.

*, ** Significant at $P<0.05$ and $<0.01$, respectively.

NS $=$ nonsignificant.

\section{Discussion}

In the present study, alkalinity in irrigation water reduced the growth of lisianthus; however, plants supplemented with an increased concentration of $\mathrm{Ca}$ at alkalinity levels from 4 to $7 \mathrm{meq} \cdot \mathrm{L}^{-1}$ of $\mathrm{HCO}_{3}{ }^{-}$exhibited improved growth and DM accumulation or were not detrimentally affected, demonstrating that $\mathrm{Ca}$ contributes to the increase of the tolerance of lisianthus to alkalinity. Alkalinity levels from 4 to $7 \mathrm{meq} \cdot \mathrm{L}^{-1}$ of $\mathrm{HCO}_{3}^{-}$have been previously demonstrated to be detrimental to several ornamental species, including roses (Rosa sp. L.), chrysanthemum (Chrysanthemum morifolium Ramat), ivy geranium [Pelargonium peltatum (L.) L'Hér.], and some cultivars of hibiscus (Hibiscus rosa-sinensis L.) (Valdez-Aguilar and Reed, 2007).
Photosynthetic rate decreased in plants exposed to increased alkalinity. Supplementary $\mathrm{Ca}$ did not ameliorate photosynthetic rate. Supplementary $\mathrm{Ca}$ did maintain a high $g_{\mathrm{S}}$ and transpiration rate when alkalinity was at $4 \mathrm{meq} \cdot \mathrm{L}^{-1}$, which may explain the lower $\psi_{\mathrm{w}}$ in young leaves. In plants irrigated with solutions containing supplementary $\mathrm{Ca}$, a higher total DM was associated with a higher $g_{\mathrm{S}}$; however, when conductance was higher than 


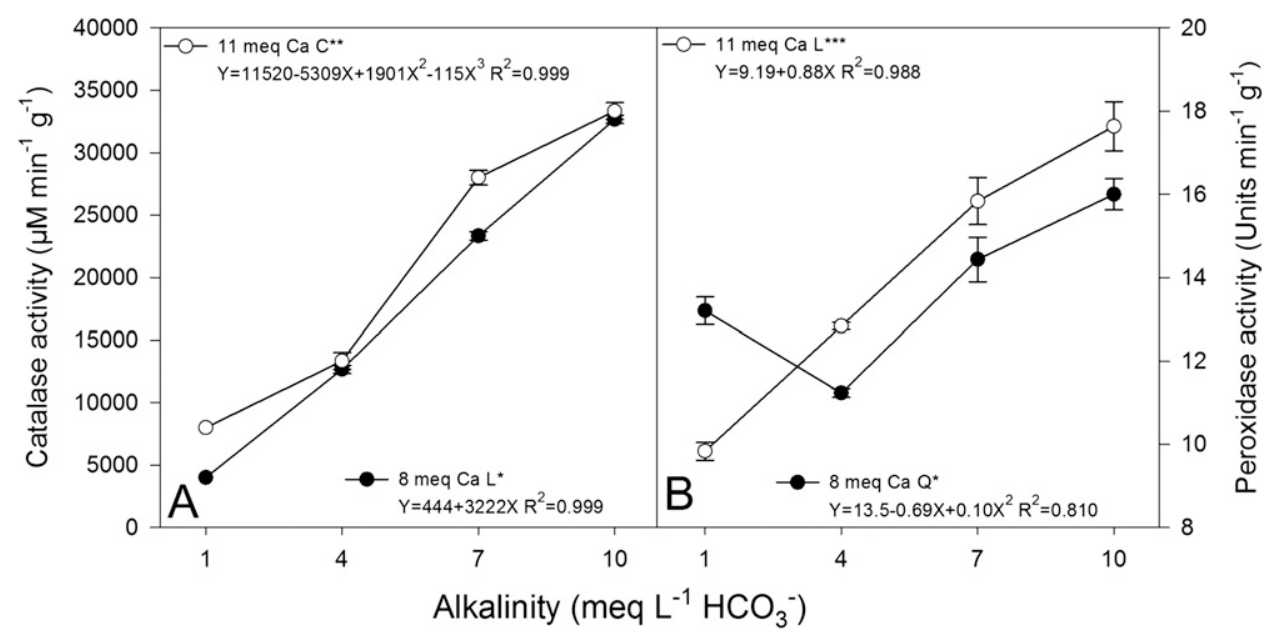

Fig. 2. (A-B) Effect of supplementary calcium $(\mathrm{Ca})$ and bicarbonate $\left(\mathrm{HCO}_{3}^{-}\right)$-induced alkalinity in the nutrient solution on catalase and peroxidase activities in lisianthus [Eustoma grandiflorum (Raf.) Shinn.] cv. ABC Blue at experiment termination ( $90 \mathrm{~d}$ after transplanting). Bars represent the SEM ( $\mathrm{n}=3$ ). L, Q, C, linear, quadratic, cubic trends, respectively; ${ }^{*}, * * * * *$ significant at $P<0.05,<0.01$, and $<0.001$, respectively. Alkalinity effect significant for peroxidase and catalase activity $(P<0.001)$, calcium effect significant for catalase activity $(P=0.002)$, and interaction effect significant for peroxidase activity $(P=0.018)$.

Table 4. Effect of calcium (Ca) concentration and bicarbonate $\left(\mathrm{HCO}_{3}{ }^{-}\right)$-induced alkalinity in the nutrient solution on macronutrient concentration (mmol. $\left.\mathrm{kg}^{-1}\right)$ (mean $\pm \mathrm{sE}, \mathrm{n}=3$ ) in shoots of lisianthus [Eustoma grandiflorum (Raf.) Shinn.] cv. ABC Blue at experiment termination ( $90 \mathrm{~d}$ after transplanting).

\begin{tabular}{|c|c|c|c|c|c|c|c|c|c|c|}
\hline & \multicolumn{2}{|c|}{ Nitrogen } & \multicolumn{2}{|c|}{ Phosphorus } & \multicolumn{2}{|c|}{ Potassium } & \multicolumn{2}{|c|}{ Calcium } & \multicolumn{2}{|c|}{ Magnesium } \\
\hline & \multicolumn{10}{|c|}{$\mathrm{Ca}\left(\mathrm{meq} \cdot \mathrm{L}^{-1}\right)$} \\
\hline$\frac{\mathrm{HCO}_{3}{ }^{-}\left(\mathrm{meq} \cdot \mathrm{L}^{-1}\right)}{1}$ & $1090 \pm 110$ & $1299 \pm 118$ & $31.8 \pm 1.3$ & $33.3 \pm 3.2$ & $516 \pm 18$ & $545 \pm 47$ & $36.2 \pm 1.5$ & $39.9 \pm 7.0$ & $117 \pm 14$ & $122 \pm 20$ \\
\hline 7 & $1333 \pm 101$ & $1165 \pm 66$ & $34.3 \pm 1.9$ & $29.7 \pm 4.0$ & $413 \pm 21$ & $385 \pm 34$ & $29.0 \pm 5.0$ & $28.3 \pm 3.2$ & $102 \pm 12$ & $93 \pm 13$ \\
\hline 10 & $1098 \pm 113$ & $956 \pm 25$ & $27.6 \pm 3.7$ & $24.7 \pm 1.9$ & $343 \pm 24$ & $342 \pm 20$ & $26.7 \pm 3.4$ & $25.2 \pm 1.7$ & $100 \pm 7$ & $82 \pm 3$ \\
\hline Trend analysis ${ }^{z}$ & $\mathrm{Q}^{*}$ & NS & NS & NS & $\mathrm{L}^{*}$ & $\mathrm{~L}^{* *}$ & $\mathrm{~L}^{* *}$ & $\mathrm{~L}^{*}$ & NS & NS \\
\hline $\mathrm{HCO}_{3}^{-}$ & \multicolumn{2}{|l|}{ NS } & \multicolumn{2}{|l|}{ NS } & \multicolumn{2}{|c|}{$P<0.001$} & \multicolumn{2}{|c|}{$P=0.004$} & \multicolumn{2}{|l|}{ NS } \\
\hline $\mathrm{Ca}$ & \multirow{2}{*}{\multicolumn{2}{|c|}{$\begin{array}{l}\text { NS } \\
\text { NS }\end{array}$}} & \multirow{2}{*}{\multicolumn{2}{|c|}{$\begin{array}{l}\text { NS } \\
\text { NS }\end{array}$}} & \multirow{2}{*}{\multicolumn{2}{|c|}{$\begin{array}{l}\text { NS } \\
\text { NS }\end{array}$}} & \multirow{2}{*}{\multicolumn{2}{|c|}{$\begin{array}{l}\text { NS } \\
\text { NS }\end{array}$}} & \multirow{2}{*}{\multicolumn{2}{|c|}{$\begin{array}{l}\text { NS } \\
\text { NS }\end{array}$}} \\
\hline Interaction & & & & & & & & & & \\
\hline
\end{tabular}

${ }^{\mathrm{z}} \mathrm{L}, \mathrm{Q}$, linear, quadratic trend, respectively.

NS, *, ** Nonsignificant and significant at $P<0.05$ and $<0.01$, respectively.

Table 5. Effect of calcium $(\mathrm{Ca})$ concentration and bicarbonate $\left(\mathrm{HCO}_{3}^{-}\right)$-induced alkalinity in the nutrient solution on micronutrient concentration $\left(\mu \mathrm{g} \cdot \mathrm{g}^{-1}\right)(\mathrm{mean} \pm$ $\mathrm{SE}, \mathrm{n}=3$ ) in shoots of lisianthus [Eustoma grandiflorum (Raf.) Shinn.] cv. ABC Blue at experiment termination (90 d after transplanting).

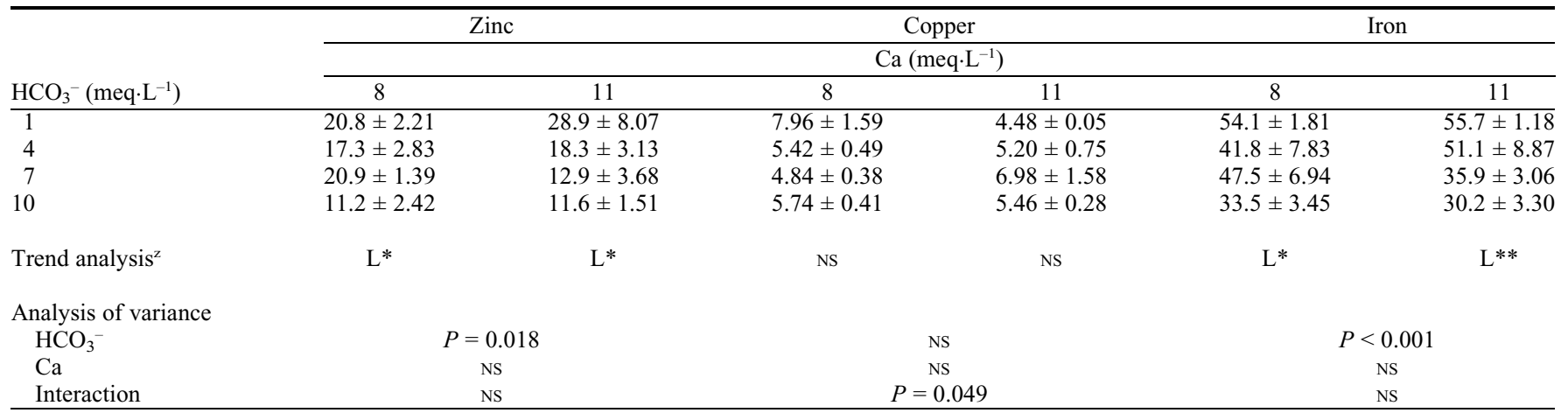

${ }^{\mathrm{z}} \mathrm{L}$, linear trend.

Ns, * ** Nonsignificant and significant at $P<0.05$ and $<0.01$, respectively.

$0.280 \mathrm{~cm} \cdot \mathrm{s}^{-1}$, like in plants with no supplemental $\mathrm{Ca}, \mathrm{DM}$ accumulation tended to decrease.

Our results show that alkalinity is associated with plant water relations by increasing $\psi_{\mathrm{w}}$ in young leaves; however, when $8 \mathrm{meq} \cdot \mathrm{L}^{-1}$ of $\mathrm{Ca}$ was provided to the plants, there was a disruption on stomata functioning as plants showed an increase in $g_{\mathrm{S}}$ and transpiration rate that led to DM reduction. The decline in functioning of the stomatal apparatus at high alkalinity was associated with a reduction in shoot $\mathrm{K}$, an ion directly involved in stomata regulation (Marschner, 1995). In plants supplemented with higher $\mathrm{Ca}$, a decrease in shoot $\mathrm{K}$ was also detected; however, $\mathrm{Ca}$ ameliorated the uptake of $\mathrm{K}$ when alkalinity was 4 meq. $\cdot \mathrm{L}^{-1}$ by buffering the reduction in shoot $\mathrm{K}$ and $\mathrm{Ca}$. Probably, extracellular $\mathrm{Ca}$ may have maintained $\mathrm{K}$ content by decreasing $\mathrm{K}$ loss through the inhibition of the $\mathrm{K}$ outward-rectified channels (Murata et al., 2000). Supplementary $\mathrm{Ca}$ and the resulting higher shoot $\mathrm{K}$ and $\mathrm{Ca}$ 
(Atkinson et al., 1990) may have interacted to regulate $g_{\mathrm{S}}$ and thus plant growth.

Chlorophyll concentration is reported to decrease with increasing alkalinity, which has been associated with a decrease in $\mathrm{Fe}$ concentration (Roosta, 2011); these findings were corroborated in our experiment. The decline in $\mathrm{Fe}$ in the shoot may have been caused by the high $\mathrm{pH}$ associated with alkalinity, which renders insoluble forms that plants are unable to uptake (De la Guardia and Alcántara, 2002). In the present study, supplementary $\mathrm{Ca}$ increased plant tolerance to alkalinity at $4 \mathrm{meq} \cdot \mathrm{L}^{-1}$ by maintaining a high shoot $\mathrm{Fe}$ concentration and a less affected chlorophyll b concentration. Similar results have been reported in white lupine (Lupinus albus L.) (Pissaloux et al., 1995). In the present study, chlorophyll b seems to be of greater importance for lisianthus because its concentration tended to be up to $100 \%$ higher than that of chlorophyll a.

Compared with plants with control Ca con centration in the nutrient solution, the lower decrease in shoot $\mathrm{N}, \mathrm{K}, \mathrm{Ca}$, and $\mathrm{Fe}$ concentration under high alkalinity $\left(4 \mathrm{meq} \cdot \mathrm{L}^{-1}\right)$ may be the result of the maintenance of membrane integrity when supplementary Ca was supplied. Tuna et al. (2007) and Siddiqui et al. (2013) reported similar results in tomato and radish (Raphanus sativus L.) plants when exposed to high salinity and B, respectively, suggesting that the supplementary $\mathrm{Ca}$ reduced the electrolytic leakage through cell membranes. Sustaining membrane integrity with $\mathrm{Ca}$ may have permitted the normal functioning of channel and carrier proteins and other enzymes such as the Fe-reductase, which would maintain or ameliorate nutrient acquisition under alkalinity stress.

Tolerance to abiotic stress in plants has been connected with the production of reactive oxygen species (ROS) that cause lipid peroxidation in cell membranes and DNA and protein damage (Gill and Tuteja, 2010). In our study, increasing alkalinity caused an increase in catalase and peroxidase activities, indicating that lisianthus responded to alkalinity stress by enhancing enzyme activity to reduce the oxidative damage caused by
ROS. Furthermore, supplementary Ca was associated with a higher catalase and peroxidase activity, probably as a result of its role as a signaling messenger (Reddy and Reddy, 2004; Schmitz et al., 2002) under stressful conditions and providing a complementary mechanism for the tolerance of lisianthus to high alkalinity.

In conclusion, in the present study, supplementary $\mathrm{Ca}$ enhanced the tolerance of lisianthus to moderate levels of alkalinity in water ( $\left.4 \mathrm{meq} \cdot \mathrm{L}^{-1}\right)$ by maintaining 1$)$ an adequate $\left.g_{\mathrm{S}} ; 2\right)$ an adequate acquisition of $\mathrm{N}, \mathrm{K}$, $\mathrm{Ca}$, and $\mathrm{Fe}$; and 3 ) reducing lipid peroxidation by enhancing the activity of antioxidant enzymes such as peroxidase and catalase.

\section{Literature Cited}

Atkinson, C.J., T.A. Mansfield, and W.J. Davies. 1990. Does calcium in xylem sap regulate stomatal conductance? New Phytol. 116:19-27.

Cartmill, A.D., A. Alarcón, and L.A. ValdezAguilar. 2007. Arbuscular mycorrhizal fungi enhance tolerance of Rosa multiflora cv. Burr to bicarbonate in irrigation water. J. Plant Nutr. 30:1517-1540.

De la Guardia, M.D. and E. Alcántara. 2002. Bicarbonate and low iron level increase root to total plant weight ratio in olive and peach rootstock. J. Plant Nutr. 25:1021-1032.

Epstein, E. 1998. How calcium enhances plant salt tolerance. Science 40:1906-1907.

FAO. 2000. Manual on integrated soil management and conservation practices. FAO Land and Water Bulletin No. 8. FAO, Rome, Italy.

Gill, S.S. and N. Tuteja. 2010. Reactive oxygen species and antioxidant machinery in abiotic stress tolerance in crop plants. Plant Physiol. Biochem. 48:909-930.

Hassan, Z. and M.G.M. Aarts. 2011. Opportunities and feasibilities for biotechnological improvement of $\mathrm{Zn}, \mathrm{Cd}$ or Ni tolerance and accumulation in plants. Environ. Exp. Bot. 72:53-63.

Jeffrey, S.W. and G.F. Humphrey. 1975. New spectrophotometric equations for determining chlorophyll a, b c1 and c2 in higher plants, algae and natural phytoplankton. Biochem Physiol. Pflanz. 167:191-194.

Kar, M. and D. Mishra. 1976. Catalase, peroxidase and polifenoloxidase activities during rice leaf. Plant Physiol. 57:315-319.

Kaya, C., H. Kirnak, D. Higgs, and K. Saltali. 2002. Supplementary calcium enhances plant growth and fruit yield in strawberry cultivars grown at high $(\mathrm{NaCl})$ salinity. Sci. Hort. 93:65-74.

Marschner, H. 1995. Mineral nutrition of higher plants. 2nd Ed. Academic Press, London, UK.

Masia, A. 1998. Superoxide dismutase and catalase activities in apple fruit during ripening and post-harvest and special reference to ethylene. Physiol. Plant. 104:668-672.

Murata, Y., S. Katsura, I. Obi, and T. Kakutani. 2000. Alterations in $\mathrm{Ca}^{2+}$ binding on plasma membrane after adaptation to salt stress of tobacco cells in suspension. Plant Cell Physiol. 41:1286-1292.

Pissaloux, A., P. Morard, and G. Bertoni. 1995. Alkalinity-bicarbonate-calcium effects on iron chlorosis in white lupine in soilless culture. Developments Plant Soil Sci. 59:127-133.

Reddy, V.S. and A.S.N. Reddy. 2004. Proteomics of calcium-signaling components in plants. Phytochemistry 65:1745-1776.

Roosta, H.R. 2011. Interaction between water alkalinity and nutrient solution $\mathrm{pH}$ on the vegetative growth, chlorophyll fluorescence and leaf magnesium, iron, manganese, and zinc concentrations in lettuce. J. Plant Nutr. 34:717-731.

Schmitz, E.M., R. Haefs, and G. Noga. 2002. Calcium deficiency-Influence on the antioxidative defense system in tomato plants. J. Plant Physiol. 159:733-742.

Siddiqui, M.H., M.H. Al-Whaibi, A.M. Sakran, H.M. Ali, M.O. Basalah, M. Faisal, A. Alatar, and A.A. Al-Amri. 2013. Calcium-Induced amelioration of boron toxicity in radish. J. Plant Growth Regul. 32:61-71.

Steiner, A.A. 1984. The universal nutrient solution. Proc. 6th Int. Congr. Soilless Cult. p. 633-649.

Steinhorst, L. and J. Kudla. 2013. Calcium and reactive oxygen species rule the waves of signaling. Plant Physiol. 163:471-485.

Tuna, A.L., C. Kaya, M. Ashraf, H. Altunlu, I. Yokas, and B. Yagmur. 2007. The effects of calcium sulphate on growth, membrane stability and nutrient uptake of tomato plants grown under salt stress. Environ. Exp. Bot. 59:173-178.

Valdez-Aguilar, L.A., C.M. Grieve, and J. Poss. 2013. Response of lisianthus to irrigation with saline water: Plant growth. J. Plant Nutr. 36: 1605-1614

Valdez-Aguilar, L.A. and D.W. Reed. 2007. Response of selected greenhouse ornamental plants to alkalinity in irrigation water. J. Plant Nutr. 30:441-452.

Zhu, J.K. 2001. Plant salt tolerance. Trends Plant Sci. 6:66-71 